\title{
Effect of Graphene Oxide and Multi-Walled Carbon Nanotubes on the Structure and Properties of Pitch Derived Carbon Foam composites
}

Amjad Hussain ${ }^{1}$, Muhammad Khan ${ }^{* 1,2}$, Ammara Nawaz', Li Tiehu' ${ }^{2}$,Amir Zada ${ }^{7}$, Hidayat Hussain ${ }^{3}$, Muhammad Imran $^{4}$, Nusrat Hussain ${ }^{5}$, Asif Hayat $^{8}$, Ataf Ali Altaf ${ }^{1}$, Ikram, Ahmad ${ }^{6}$, Hassan Fouad ${ }^{9}$, Yaser H.A. Elewa ${ }^{10,11}$, Gaber El-Saber Batiha ${ }^{12}$

1. ${ }^{I}$ Department of Chemistry, University of Okara, Okara, Pakistan.

2. $\quad{ }^{2}$ School of Materials Science and Engineering, Northwestern Polytechnical University, Xian, Shaanxi, P.R.China.

3. $\quad{ }^{3}$ Leibniz Institute of Plant Biochemistry, Department of Bioorganic Chemistry, Weinberg 3, D-06120 Halle (Salle), Germany

4. $\quad{ }^{4}$ Department of Chemistry, Faculty of Science, King Khalid University, P.O. Box 9004, Abha 61413, Saudi Arabia.

5. $\quad{ }^{5}$ Department of Chemistry, University of Baltistan Skardu, Skardu-16100, Pakistan.

6. ${ }^{6}$ Department of Chemistry, University of Sahiwal, Sahiwal-57000, Punjab, Pakistan

7. $\quad{ }^{7}$ Department of Chemistry, Abdul Wali Khan University, Mardan, K.P.K, Pakistan.

8. $\quad{ }^{8}$ College of Chemistry, Fuzhou University, Fuzhou, P.R.China.

9. Applied Medical Science Department, Community College, King Saud University, P.O Box 10219, Riyadh 11433, Saudi Arabia

10. Department of Histology and Cytology, Faculty of Veterinary Medicine, Zagazig University, Zagazig 44519, Egypt

11. Laboratory of Anatomy, Department of Biomedical Sciences, Graduate School of Veterinary Medicine, Hokkaido University, Sapporo 060-0818, Hokkaido, Japan

12. Department of Pharmacology and Therapeutics, Faculty of Veterinary Medicine, Damanhour University, Damanhour 22511, AlBeheira, Egypt Corresponding Author: Muhammad Khan mkhanchemistry@yahoo.com

\begin{abstract}
Multi-walled carbon nanotubes (MWCNTs) and graphene oxide (GO) reinforced carbon foam (CF) composite were prepared by direct pyrolysis of MWCNTs, GO and mesophase coal tar pitch. The effect of additive amount of the mixture of MWCNTs and GO on the microstruture and properties of carbon foam was analzyed by transmission electron miscroscopy (TEM), scanning electron microscopy (SEM), X-ray diffraction (XRD), Four-probe resistance meter, universal testing machine, and laser thermal conductivity tester respectively. The result shows that MWCNTs and GO had significant impact on the microstructure of carbon foam. Futhermore, the electrical, mechanical and thermal properties of carbon foam composites were significantly enhanced by increasing the additive amount. Maximum compressive strenght of $19.2 \mathrm{MPa}$ and Young's modulus of $56.8 \mathrm{MPa}$ of CF composite were observed. Similarly, Highest thermal conductivity of $30.91 \mathrm{~W} / \mathrm{m} . \mathrm{K}$ and electrical conductivity of $27.2 \times 10^{3} \mathrm{~S} / \mathrm{m}$ were observed at $2 \mathrm{wt}$. $\%$ of MWCNTs-GO additive loading.
\end{abstract}

Key Words: Carbon foam, multi walled carbon nanotubes, Graphene oxide, electrical, mechanical and thermal properties.

\section{Introduction}

Graphene are two dimensional (2D) allotrope of carbon, having a single layer of carbon atoms arranged in hexagonal pattern. It is well known material due to its characteristic electrical and mechanical properties. Moreover, graphene has versatile applications in electrochemicalelectrodes, solar cells, sensor and many other materials. Graphene can be synthesized by various methods such as, micro chemical exfoliation of graphite, solvo-thermal synthesis, reduction of graphene oxide, epitaxial growth on electrically insulating surface, and chemical vapor deposition. In addition, currently 3D graphene can be prepared by chemical vapor deposition and show better conductivity. It shows better electrochemical properties due to large surface area ${ }^{[1,2]}$. While graphene oxide (GO) is amphiphilic molecule having both aliphatic and aromatic structural 
arrangement. In addition, GO exhibits both hydrophilic and hydrophobic properties. Hydrophilicity of GO is due to of the presence of oxidized $\mathrm{Sp}^{3}$ carbon atom in the aliphatic region. While hydrophobicity is because of aromatic region having unoxidized benzene rings having $\mathrm{Sp}^{2}$ carbon atoms. GO demonstrated chemical functionalization and high dispersion due to oxygen containing functional groups. Due to $\pi-\pi$ supramolecular interaction, the aromatic region provides active site for the interactions with other molecules. Because of these intriguing chemical and physical properties, GO is used to prepare composite with other nanostructures ${ }^{[3]}$. Similarly, Carbon nanotubes are one dimensional (1D) allotropes of carbon having excellent electrical, mechanical and thermal properties. Multi-walled carbon nanotubes have many layer of graphene. Carbon nanotubes have many applications in energy storage and conversion devices ${ }^{[2]}$. Both these nanoparticles show characteristic properties in Nano composite.

Carbon foam (CF) is a porous spongy material consisting of interconnected network of atoms. It has many attractive properties, such as, light weight, low density, low cost, chemical inertness, high surface area, high thermal stability, adjustable electrical, thermal conductivity, high strength and corrosion resistance ${ }^{[4,5]}$. Due to these properties CF have many tremendous applications including oil removal, catalyst support, electromagnetic shielding, as electrode in electrochemical cells, energy storage, water purification, radar absorption-filter, heat exchanger, and as matrix for sensor ${ }^{[6,7]}$. Wang et al. examined the effect of clay on the mechanical and thermal conductivity of CF. The outcome showed that when clay is mixed with CF, the mechanical strength of CF increases but thermal conductivity decreases by addition of clay ${ }^{[8]}$. Moreover, Chen et al. prepared $\mathrm{CF}$ by the direct carbonization of melamine foam. He indicated that CF shows low mechanical properties, but desirable electrical conductivities as well as high surface area and high porosity ${ }^{[4]}$. In another report, Liu et al. studied the effect of MWCNTs on the property of pitch derived $\mathrm{CF}$ and found that the electrical conductivity of CF increases with increasing MWCNTs but the thermal conductivity first increases then decreases ${ }^{[9]}$. Kim et al. synthesized CF from carboxymethyl cellulose (CMC) and Ag, $\mathrm{Al}$ and carbon nanotubes (CNTs), also graphene was added in the foam, then studied the effects of these on the thermal conductivity of CF [10]. Kumar et al., grown MWCNTs on CF by using the method of chemical vapor deposition and found that thermal conductivity of CF rised by $75 \%$ due to the addition of MWCNTs ${ }^{[11]}$. In another report, Kumar et al. studied the effect of nanosized iron particles from ferrocene on CF prepared from coal tar pitches and observe that nanosized iron particles work as a catalyst and there is better graphitization of CF. They further observed that the thermal and electrical properties as well as electromagnetic interference shielding effectiveness increases with increasing nanosized iron particles ${ }^{[12,34]}$. Yu et al. synthesized ultralight flexible CF from melamine foam using the method of carbonization. They further investigated the effect of temperature on the porosity and thermal property of ultralight flexible CF. They observed that with increasing temperature, the pore diameter as well as thermal properties are badly affecting. With increases pore diameter thermal conductivity increases, so better results could be achieved by modifying the structure ${ }^{[13] \text {. }}$

Yadav et al. synthesized high thermal conducting CF by dissolving water slurries of different concentration of mesophase pitches using a template of polyurethanes foam and graphitization is done with the help of different heat treatment. They observed that graphitized CF show better pore structure and excellent thermal conductivity up to $60 \mathrm{~W} / \mathrm{m} \mathrm{K}$. It also show double specific thermal conductivity compare to copper [14]. Zhang et al. synthesized ultralight CF for achieving high mechanical strength and electromagnetic interference shielding effectiveness by carbonization of phthalonitriles-based polymer. He found that the obtained $\mathrm{CF}$ had high compressive strength, low density and high performance ${ }^{[15]}$. Peng et al. worked to achieve better 
performance of supercapacitor based on ultralight and elastic three dimensional (3D) porous melamine foam (MF)-derived macroporous carbon (3DPMFDMC)/reduced graphene oxide (rGO)/polyaniline (PANI) nanocomposites (denoted as 3DPMFDMC/rGO/PANI) nanocomposites. The 3D MFDMC/ rGO prepared by using the method of carbonization at high temperature and $\mathrm{MF}$ immersed in $\mathrm{GO}$ and $\mathrm{GO}$ reduce. By chemical oxidation polymerization, polyaniline disperse on 3D MPDMC/rGO. They observed that nanocomposite show high electrical conductivity, large surface area, high performance and high efficiency ${ }^{[16,35]}$. Meng et al. prepared $\mathrm{CF}$ using the $\mathrm{Ni}$ as a template and $\mathrm{CF}$ deposited on Ni-template. They observe that certain properties of $\mathrm{CF}$ enhanced by this method such as porosity, high thermal conductivity and high electrical conductivities ${ }^{[17]}$. Moreover, Caicedo et al. used the method of blow foaming to synthesize CF from pyroligneous acids, obtained from angustifolia Kunth and CF studied at different carbonization temperatures, and mechanical property investigate by stress strain test, which indicated that CF have high mechanical property ${ }^{[18]}$. All these Nano particles enhanced one property but at the same time badly affected other properties. More work is required to get the desirable properties.

In this study we have incorporated the various ratios of GO and MWCNTs as additives in mesophase pitches to investigate the effect of GO and MWCNTs on the microstructure of CF composites. The fabrication and reinforcement effect of these additives were further checked in terms of mechanical, thermal and electrical, properties of CF composites.

\section{Experiment}

\subsection{Material}

The carbon foam was prepared using coal tar pitches that were provided by Wuhan Steel Corporation limited, China. The physical characteristics of coal tar pitches are given in Table-1. Graphite flakes and the other chemicals used, such as, $\mathrm{KMnO}_{4}, \mathrm{H}_{2} \mathrm{SO}_{4}, \mathrm{H}_{3} \mathrm{PO}_{4}, \mathrm{HCl}, \mathrm{H}_{2} \mathrm{O}_{2},\left(\mathrm{H}_{3} \mathrm{C}\right)_{2}$ $\mathrm{CO}$ and $\mathrm{C}_{2} \mathrm{H}_{5} \mathrm{OH},\left(\mathrm{C}_{2} \mathrm{H}_{5}\right)_{2} \mathrm{O}$ were purchase from Sigma Aldrich (Pvt.) Ltd., Australia. Multiwalled Carbon nanotubes were purchased from Tsinghua University, China. Nitric acid and sulfuric acid were used for the purification of carbon nanotubes.

Table.1: Physical characteristics of commercial coal tar pitches.

\begin{tabular}{ccccc}
\hline $\begin{array}{c}\text { Soft point } \\
\left({ }^{\circ} \mathrm{C}\right)\end{array}$ & Quinoline insoluble & Benzene insoluble & C/H (atomic) & Carbon yield \\
81 & $($ wt. \%) & (wt. \%) & (ratio) & $($ wt \%) \\
\hline
\end{tabular}

\subsection{Preparation of Graphene oxide}

The preparation of GO involve the oxidation of graphite flakes as the method describe by Marcano et al ${ }^{[19]}$. In this method $\mathrm{H}_{2} \mathrm{SO}_{4}$ and $\mathrm{H}_{3} \mathrm{PO}_{4}$ were used in 9:1. This mixture was mixed with graphite flakes. The KMnO4 in 6 equal portions was slowly added to this mixture. Then mixture was heated at $50^{\circ} \mathrm{C}$ for $12 \mathrm{~h}$ with continuous stirring. Furthermore, the mixture was cool to room temperature and put on ice with $3 \mathrm{ml}$ of $30 \% \mathrm{H}_{2} \mathrm{O}_{2}$. Then the mixture was passed through 
a metal U.S. Standard Testing Sieve, and then filtered through polyester fiber mixture. The centrifugation of filtrate was done, then by using $200 \mathrm{~mL}$ of water, $200 \mathrm{~mL}$ of ethanol, and 200 $\mathrm{mL}$ of $30 \% \mathrm{HCl}$ solid material washed. After every wash mixture passes through U.S. Standard Testing Sieve and filtered by polyester fiber. The mixture obtained so was coagulated by using $200 \mathrm{~mL}$ ether and filter by using PTFE membrane. The material dried under high vacuum for an overnight ${ }^{[19]}$.

\subsection{Purification of MWCNTs}

For the purification of MWCNTs concentrated sulfuric acid and nitric acid were used, in ratio of $3: 1$ by volume. The MWCNTs was added into mixture of concentrated nitric and sulfuric acid solution. The slurry was mixed by stirring it through ultrasonic machine at $60^{\circ} \mathrm{C}$ for 8 hours. The MWCNTs was then thoroughly washed through deionized water until the $\mathrm{pH}-7$ was obtained. The dried MWCNTs resulted after filtration and kept at $90^{\circ} \mathrm{C}$ for 10 hours ${ }^{[9]}$.

\subsection{Preparation of carbon foam composite}

The commercial coal tar pitches were used for the preparation of CFs. First of all raw coal tar was subjected to pretreatment to form mesophase pitches. For this purpose about $120 \mathrm{~g}$ of coal tar was taken and grounded to fine powder to get a particle of $70 \mu \mathrm{m}$, then heated to $430^{\circ} \mathrm{C}$ for 5 $\mathrm{h}$ in nitrogen atmosphere. The pretreated pitches were again heated and grounded to fine powder ${ }^{[20]}$. Then CF composite was prepared by adding different amount of GO and MWCNTs in CF. The slurry of pretreated pitches and nanoparticles was dissolved in ethanol, so that nanoparticles evenly dispersed with pretreated pitches. To remove ethanol, mixture was heated to $80^{\circ} \mathrm{C}$. The dried mass obtained was grounded to obtain $70 \mu \mathrm{m}$ sizes. Then by adding different amounts of nanoparticles, composite of CF-MWCNTs and CF-MWCNTs-GO were prepared. The blank sample containing only CF was also prepared. For measuring properties, the 1, 2, $4 \mathrm{wt} \%$ of MWCNTs were mixed in pretreated pitches to get MWCNTs-CF composite. While for preparing CF-MWCNTs-GO, the MWCNTs and GO were added in additive amounts to the pretreated pitches to form 1,2 , and $4 \mathrm{wt} \%$ composites. In reaction vessel containing $3 \mathrm{MPa}$ nitrogenic atmospheres, foaming of MWCNTs-GO-pretreated pitches was carried out. The mixture was heated up to $500^{\circ} \mathrm{C}$ for $2 \mathrm{~h}$. Then the process of carbonization at $850^{\circ} \mathrm{C}$ and graphitization at $2400^{\circ}$ $\mathrm{C}$ of $\mathrm{CF}$ containing nanoparticles was carried out in nitrogenic atmosphere for $2 \mathrm{~h}$ and $1 \mathrm{~h}$ respectively, with heating rate of $2^{\circ} \mathrm{C}$ per minute. $\mathrm{CF}$ containing MWCNTs and GO as nanoparticles were obtained cooled and then characterized.

\section{Characterization}

To investigate the microstructure and morphology of CF containing GO and MWCNTs, Transmission Electron Microscopy (TEM) (Hitachi H-600) was used. The Scanning Electron Microscopy (SEM) (XL30) was used to investigate the cell structure, size of cell and also to investigate the dispersion of nanoparticles in CF. To analyze the crystal-structure information of CF sample X-ray diffractometer (XRD) (D/Max $2500 \mathrm{~V}$ PC-1, Cu-Ka radiation) was used with the scanning rate of $2 / \mathrm{min}$. Mechanical properties, such as, compressive strength and young's

modulus were measured by Universal Testing Machine having crosshead speed of $5 \mathrm{~mm} \mathrm{~min}^{-1}$ and 
load cell of 100 N. Sample was compressed between two stainless plates. The compressive yield strength $(\sigma)$ was calculated by using the following equation:

$$
\sigma=\mathrm{F} / \mathrm{A} \quad \text { Eq. (1) }
$$

In this equation "F" is the load at yield and "A" is area of cross section. Similarly, thermal conductivity was measured by Netzsch LFA 457 conductivity tester, which uses laser flash technique. Test were performed between 25 and $900^{\circ} \mathrm{C}$. Thermal conductivity $(\lambda)$ was measured by following equation

$$
\lambda=\rho \mathrm{C} \alpha \quad \text { Eq. (2) }
$$

Where " $\rho$ " is the sample density, " $C$ " is heat capacity and " $\boldsymbol{\alpha}$ " is the thermal diffusivity. The electrical conductivity was measured by using four-probe resistance meter (SX 1944, china). The room temperature electrical conductivity was calculated by taking reciprocal of electrical resistivity. And electrical resistivity was measured by four-probe method. The both sides of sample were measured and two measured values were averaged.

\section{Result and discussion}

\subsection{Transmission Electron Microscopy}

Transmission electron microscopy was used to investigate the particles size and their average range. For removing impurities on MWCNTs, acid treatment was performed. Fig-1a shows the TEM result of purified MWCNTs. Due to impurities present on CNTs there are clusters of MWCNTs that reduce after acid treatment and fine arrangement of nanofiller obtained in nanometer range. Fig-1b shows hybrid composite of GO and MWCNTs in CF. TEM result shows that GO and MWCNTs are uniformly dispersed in CF. This uniform dispersion of GO and MWCNTs have great impact on the properties of CF ${ }^{[9]}$.
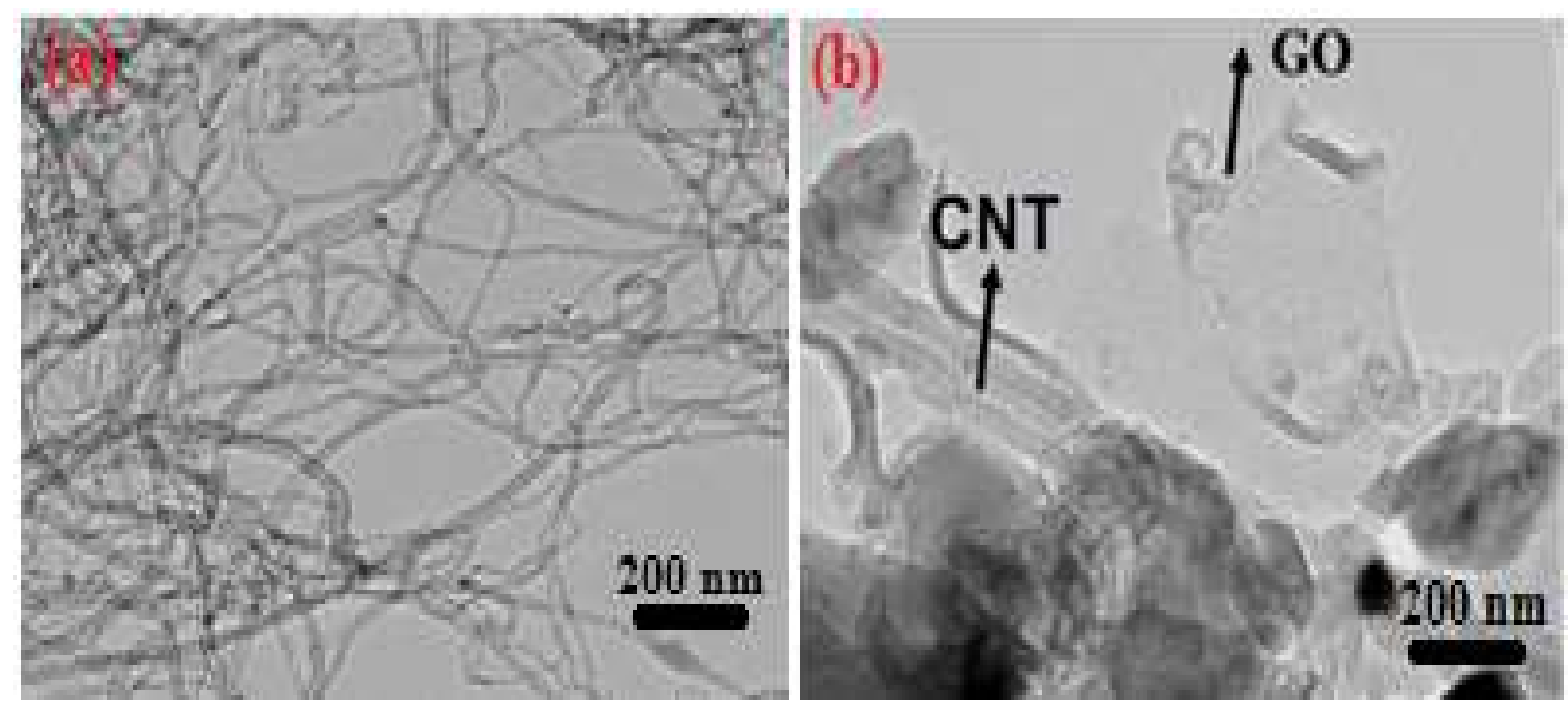

Fig-1: TEM results of purified MWCNTs (a) and hybrid MWCNTs and GO dispersed CF composite (b). 


\subsection{Scanning Electron Microscopy}

The scanning electron microscopy was used to examine the morphology of CF and the dispersion of nanoparticles in CF. Fig-2 Shows the SEM images of CF (a) and SEM images of CF after addition of GO and MWCNTs (b). Fig-2a shows the CF without any nanoparticles. Cell size and structure of CF are not uniform in pure CF. Fig-2b, c, $\mathbf{d}$ shows the SEM images of 1, 2 and 4 $\mathrm{w} \%$ of MWCNTs in CF. It can be observed that after the addition of MWCNTs in CF, cell size and structure becomes more organized and uniform. The MWCNTs act as cracks barrier that lead to reduction of cracks in structure of CF. Fig-2e, $\mathbf{f}, \mathbf{g}$ shows the 1,2,4 wt \% CF/MWCNTs-GO hybrid composite. It can be seen that prominent changes occurs in microstructure of $\mathrm{CF}$ after the addition of MWCNTs and GO in CF. Due to uniform and homogeneous dispersion of MWCNTs and $\mathrm{GO}$ in $\mathrm{CF}$, cellular structure become more uniform and spherical, also imperfections greatly reduced. The reason behind this, is the reduction of viscosity of pretreated pitches during foaming process ${ }^{[33]}$. When MWCNTs and GO introduce in CF viscosity of pretreated pitches reduced at the foaming temperature, as a result bubble easily grows, combine and uniform CF structure form. SEM results shows that MWCNTs and GO greatly improved the morphological behavior of CF such as cellular structure, porosity, size and well organize homogeneity $[9,20]$. 

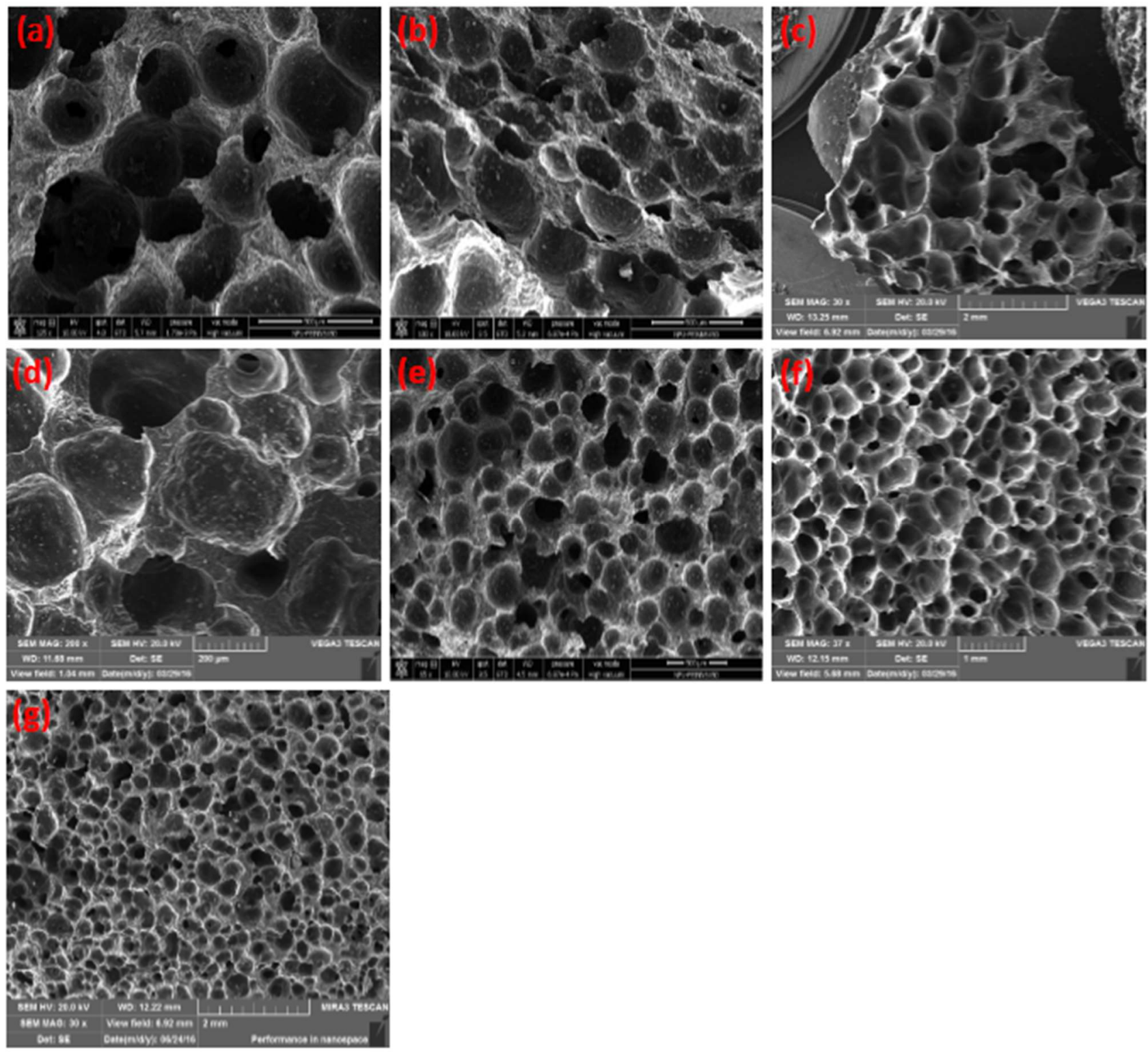

Fig-2: SEM images of pure CF (a), 1, 2, 4 wt. \% CF/MWCNTs (b, c, d), while 1, 2 and 4 wt. \% CF/MWCNTs-GO hybrid composites (e, f, g).

\subsection{X-ray Diffraction}

The XRD technique was used to examine the crystal structure, phase, purity and peak shift upon the formation of hybrid composite. Fig-3 shows the XRD Spectra of pure CF, CF/ MWCNTs and CF/MWCNTs/GO. Pure CF shows peak $27.5^{\circ}, 44.5^{\circ}, 56.3^{\circ}, 78.5^{\circ}$ corresponding to planes 002 , 101, 004, 110 which confirm the crystalline structure of CF. The XRD of CF/MWCNTs shows more intense peak which shows that the crystal structure much more improved than pure CF [21]. While CF/MWCNTs/GO shows high intensity peak which shows MWCNTs and GO more improve CF crystal structure. There is one additional peak at $11.5^{\circ}$ corresponding to plane 001 which shows the oxygen-containing functional group in $\mathrm{GO}{ }^{[22]}$. 


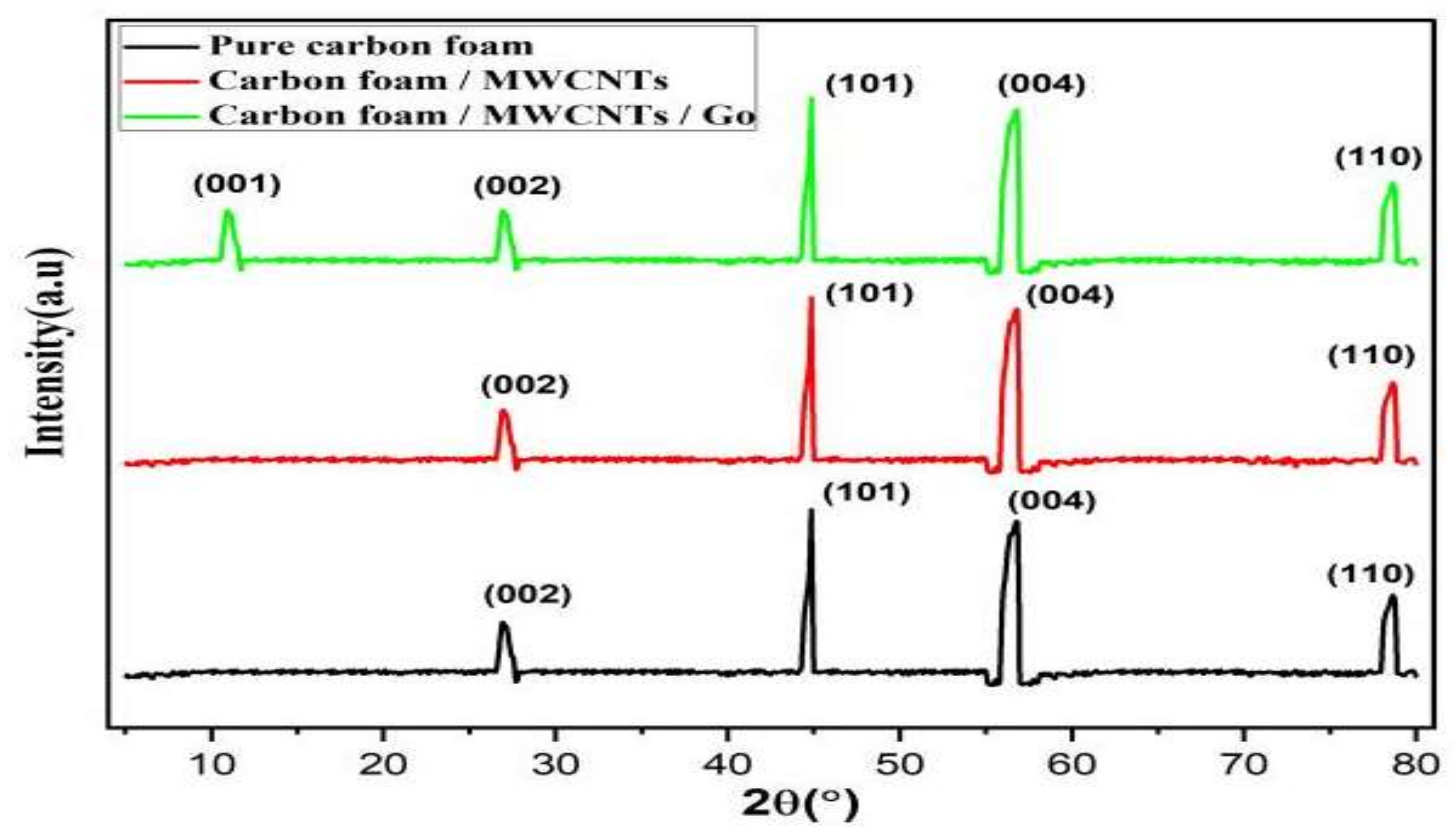

Fig-3: shows the XRD spectra of carbon foam with different amount of GO/MWCNTs.

\subsection{Mechanical properties}

The Mechanical property of CF composite containing GO and MWCNTs as additive, were observed in term of compressive strength and young's modulus. Fig-4 (a) and (b) shows the compressive strength and Young's modulus values of CF/MWCNTs and CF/MWCNTs-GO composites. It can be observed from the Fig-4(a) that compressive strength of CF becomes much improved after the addition of 1 to 4 wt. \% MWCNTs and GO. Fig-4 (a) clearly reveals that initially the compressive strength of pure $\mathrm{CF}$ was found $6.8 \mathrm{MPa}$ while after the additive amount of MWCNTs reaches up to $2 \mathrm{wt}$. \%, maximum compressive strength of $15.3 \mathrm{MPa}$ was observed. The main reason for increase of compressive strength of CF after addition MWCNTs is due to the modification of MWCNTs that hinder the propagation of cracks in cell structure and improve interfacial bonding. Moreover, further addition of MWCNTs reduces the compressive strength due to cluster formation. It can also be seen that, when both MWCNTs and GO are synergistically introduce in CF matrix maximum compressive strength of $19.2 \mathrm{MPa}$ resulted. This indicates that

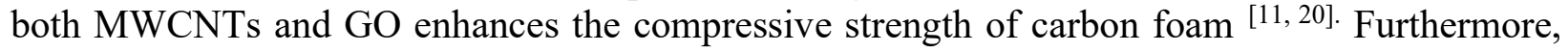
Fig-4(b) indicates the mechanical properties of CF composites containing various loading of MWCNTs and GO as additives in term of Young's modulus. The pure CF shows only $12 \mathrm{MPa}$ of Young's modulus. After the addition of MWCNTs maximum value of Young's modulus was observed and the value of $40 \mathrm{MPa}$ was observed when $2 \mathrm{wt}$. \% MWCNTs were added. After that MWCNTs and GO synergistically introduce and value reaches to $56.8 \mathrm{MPa}$ when 2 wt. \% of MWCNTs-GO were applied. This highest value of Young's modulus shows that very high energy is needed to break CF composite having MWCNTs and GO as additives ${ }^{[23,24]}$. 

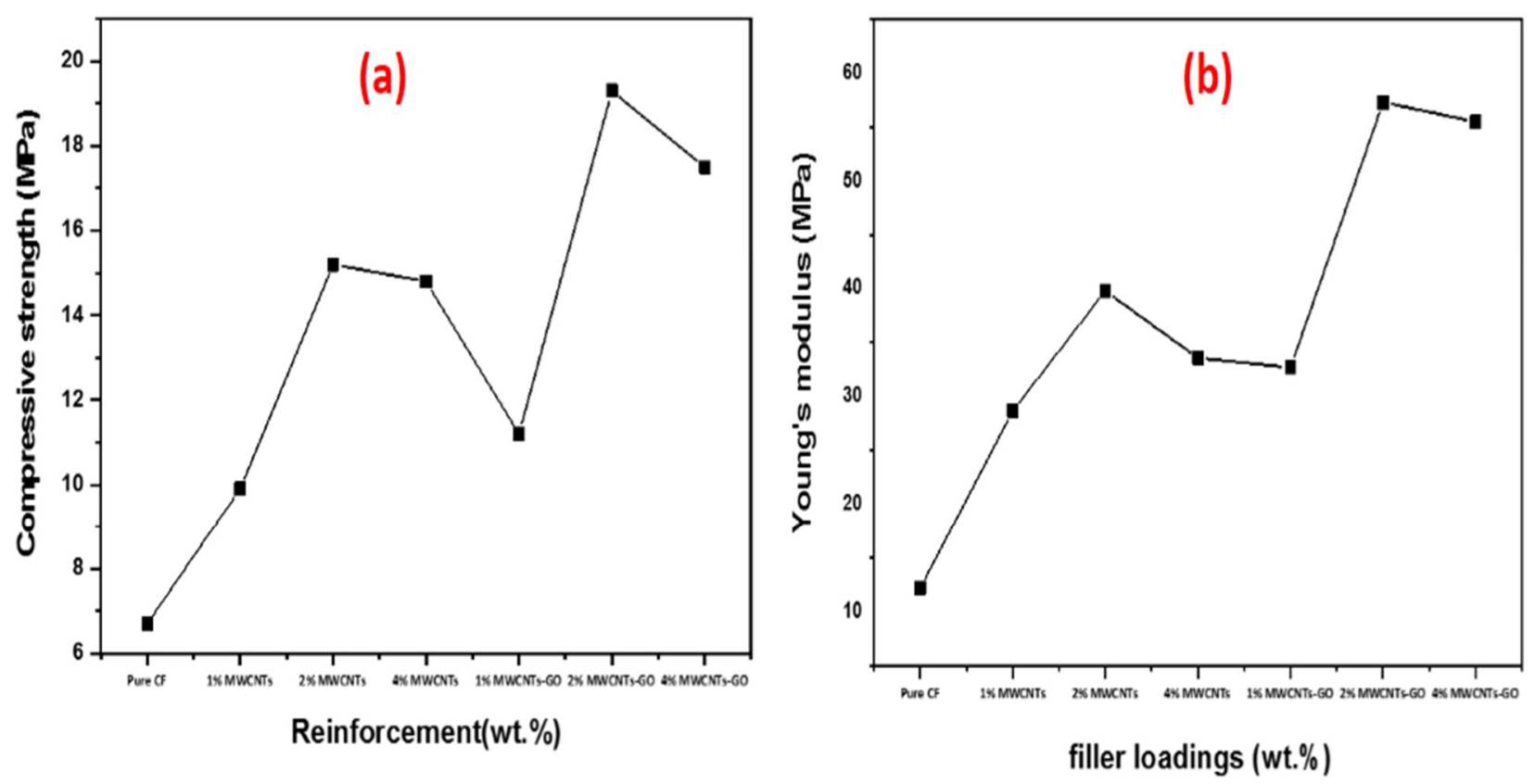

Fig-4: Compressive strength (a) and Young's modulus (b) of CF composites having variable reinforcements amount.

\subsection{Thermal properties}

Fig-5 shows the thermal conductivity of CF containing different amount of $\mathrm{GO}$ and MWCNTs as additive. As the temperature is rises up to $900{ }^{\circ} \mathrm{C}$ with loading of GO and MWCNTs the thermal conductivity of carbon foam enhances. It can be clearly observed that thermal conductivity of pure CF is low. Maximum thermal conductivity of $28.93 \mathrm{~W} / \mathrm{m} \mathrm{K}$ is observed when there is a loading of $2 \mathrm{wt} . \%$ MWCNTs at a temperature of $900{ }^{\circ} \mathrm{C}$. While $4 \mathrm{wt} . \%$ MWCNTs shows low thermal conductivity due to non-homogeneous dispersion of MWCNTs in CF. The maximum thermal conductivity of 30.91 and $29.1 \mathrm{~W} / \mathrm{m} \mathrm{K}$ was shown by CF containing 2 and $4 \mathrm{wt} \%$ of GO and MWCNTs as additives. The incorporation of GO along with MWCNTs enhance the thermal conductivity of $\mathrm{CF}$ due to the excellent thermal properties and homogeneous dispersion of nanofiller. This study shows that CF shows excellent thermal conductivity when GO is used synergistically with MWCNTs ${ }^{[25,26]}$. 


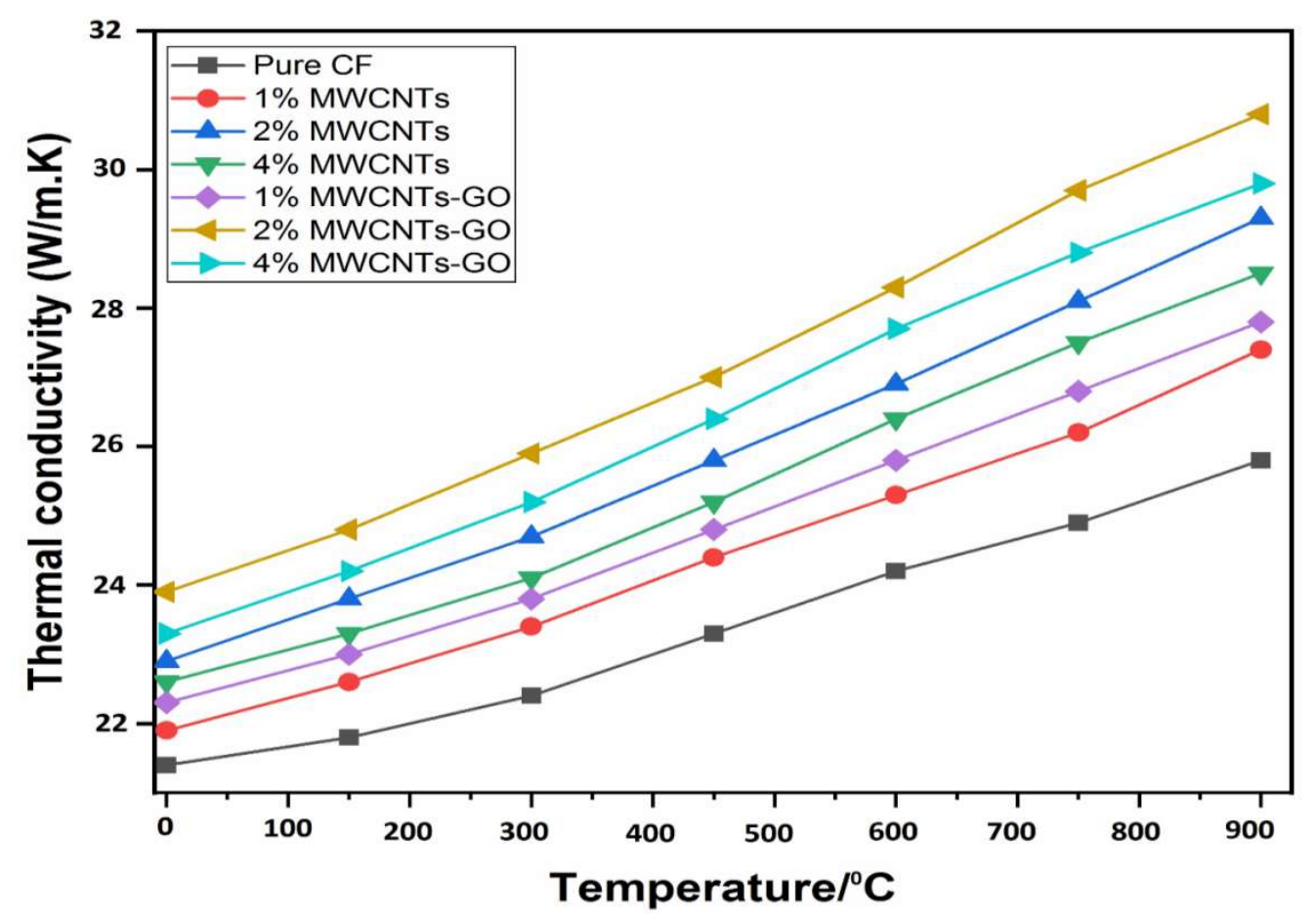

Fig-5: Thermal conductivity of CF composites with various nanofillers loadings.

\subsection{Electrical properties}

Fig-6 shows the electrical conductivity of $\mathrm{CF}$ at various concentrations of nanofiller. It can be seen that electrical conductivity of pure $\mathrm{CF}$ is $7.1 \times 10^{3} \mathrm{~S} / \mathrm{m}$ which shows that pure CF is poor conductor. After the addition of MWCNTs electrical conductivity increase to several order of magnitude. The result shows that maximum electrical conductivity of $22.1 \times 10^{3} \mathrm{~S} / \mathrm{m}$ was observed when 2 wt. \% MWCNTs added as additive amount. This enhancement in electrical conductivity is due to the conduction path of electron ${ }^{[31,32]}$. After that electrical conductivity deceases due to nonhomogeneous dispersion of MWCNTs. Fig-6 also shows that when GO added in additive amount along with MWCNTs the electrical conductivity enhanced up to $27.2 \times 10^{3} \mathrm{~S} / \mathrm{m}$ due to core structure and surface layer that function as charge transfer channel to improve electrical conductivity of $\mathrm{CF}$ composite. This electrical conductivity result indicates that consolidation of GO along with MWCNTs had much better effect on electrical properties of CF composites ${ }^{[27-30]}$. 


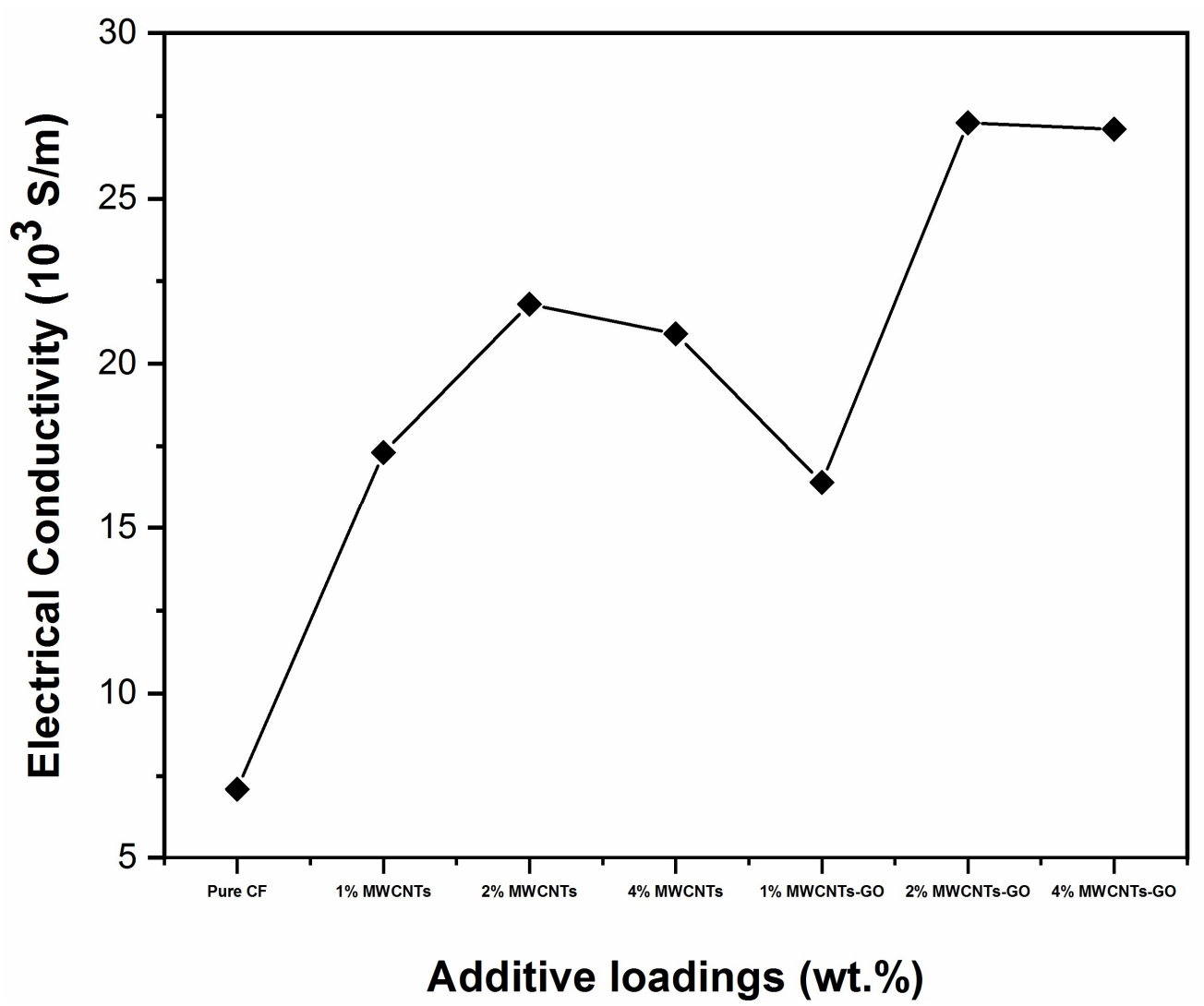

Fig-6: Electrical conductivity of CF composites at various amount of nanofillers contents.

\section{Conclusion}

In this work preparation and properties of carbon foam (CF) containing additive amount of MWCNTs and GO were studied. The microstructure investigation showed that both MWCNTs and GO has significant impact on the pore size and cell structure of carbon foam. Result revealed that electrical, mechanical and thermal properties increases gradually with increasing nanofiller additive content. The maximum compressive strength and young's modulus of 19.2 and $56.8 \mathrm{MPa}$ respectively were shown by $\mathrm{CF}$ at $2 \mathrm{wt} \% \mathrm{MWCNTs-GO}$ as additive. In addition, the highest thermal conductivity of $30.91 \mathrm{~W} / \mathrm{m} . \mathrm{K}$ at $900^{\circ} \mathrm{C}$ and electrical conductivity of $27.2 \times 10^{3} \mathrm{~S} / \mathrm{m}$ was observed at $2 \mathrm{wt} \%$ MWCNTs-GO loading.

\section{Deceleration of Interest Statement}

On behalf of all authors, the corresponding author declared that this work is the original work of authors and all standards were followed accordingly and there is no conflict of interest

\section{Acknowledgments}

This research has been Financially Supported for the Postdoctoral study by the Shaanxi Province and Natural Science Foundation of China under the (Grant No: 2016JQ5108). The co- 
author M. Imran express his appreciation to the Deanship of Scientific Research at King Khalid University Saudi Arabia for funding through research groups program under grant number R.G.P. 2/3/42 and Project number (RSP-2021/117), King Saud University, Riyadh, Saudi Arabia.

\section{References}

[1] W. Chen, L. Yan, P.R. Bangal, Preparation of graphene by the rapid and mild thermal reduction of graphene oxide induced by microwaves, Carbon, 48 (2010) 1146-1152.

[2] X. Dong, Y. Ma, G. Zhu, Y. Huang, J. Wang, M.B. C. Park, L. Wang, W. Huang, P. Chen, Synthesis of graphene-carbon nanotube hybrid foam and its use as a novel three-dimensional electrode for electrochemical sensing, J. Mater. Chem., 22 (2012) 17044-17048.

[3] V. Man, S.M. Chen, B.S Lou, Three dimensional graphene oxide-carbon nanotubes and graphene-carbon nanotubes hybrids, Int. J. Electrochem. Sci., 8 (2013) 11641-11660.

[4] S. Chen, G. He, H. Hu, S. Jin, Y. Zhou, Y. He, Y. Zhou, Y. He, S. He, F. Zhaob, H. Hou, Elastic carbon foam via direct carbonization of polymer foam for flexible electrodes and organic chemical absorption, Energy Environ. Sci., 6 (2013) 2435-2439.

[5] R.T. Woodward, F. Markoulidis, F.D. Luca, D. Malko, D.B. Anthony, T.O. McDonald, M.S.P. Shaffer, A. Bismarck, Carbon foams from emulsion-templated reduced graphene oxide polymer composites: electrodes for supercapacitor devices, J. Mater. Chem. A, 6 (2018) 18401849.

[6] H. Liu, T. Li, X. Wang, W. Zhang, T. Zhao, Preparation and characterization of carbon foams with high mechanical strength using modified coal tar pitches, J. Anal. Appl. Pyrolysis, 110 (2014) 442-447.

[7] J. Chen, J. Xu, S. Zhou, Ni. Zha, C.P. Wong, Nitrogen-doped hierarchically porous carbon foam: a free-standing electrode and mechanical support for high-performance supercapacitors, Nano Energy, 25 (2016) 193-202.

[8] X. Wang, J. Zhong, Y. Wang, M. Yu, A study of the properties of carbon foam reinforced by clay, Carbon, 44 (2006) 1560-1564.

[9] H. Liu, T. Li, T. Huang, X. Zhao, Effect of multi-walled carbon nanotube additive on the microstructure and properties of pitch-derived carbon foams, J. Mater. Sci., 50 (2015) 7583-7590. [10] H.G. Kim, Y.S. Kim, L.K. Kwac, H.J. Shin, S.O. Lee, U.S. Lee, H.K. Shin, Latent heat storage and thermal efficacy of carboxymethyl cellulose carbon foams containing Ag, Al, carbon nanotubes, and graphene in a phase change material, Nanomaterials, 9 (2019) 158-169. 
[11] R. Kumar, S.R. Dhakate, T. Gupta, P. Saini, B.P. Singh, R.B. Mathur, Effective improvement of the properties of light weight carbon foam by decoration with multi-wall carbon nanotubes, J. Mater. Chem. A, 1 (2013) 5727-5751.

[12] R. Kumar, S.R. Dhakate, P. Saini, R.B. Mathur, Improved electromagnetic interference shielding effectiveness of light weight carbon foam by ferrocene accumulation, RSC Adv., 3 (2013) 4145-4151.

[13] S. Yu, Z. Chen, Y. Wang, R. Luo, Y. Pan, A study of thermal insulation properties and microstructure of ultra-light 3D-carbon foam via direct carbonization of polymer foam, J. Porous. Mat., 25 (2017) 527-536.

[14] A. Yadav, R. Kumar, G. Bhatia, G.L. Verma, Development of mesophase pitch derived high thermal conductivity graphite foam using a template method, Carbon, 49 (2011) 3622-3630.

[15] L. Zhang, M. Liu, S. Roy, E.K. Chu, K.Y. See, X. Hu, Phthalonitrile-based carbon foam with high specific mechanical strength and superior electromagnetic interference shielding performance, ACS appl. mater. inter., 8 (2016) 7422-7430.

[16] C. Peng, J. Yu, S. Chen, L. Wang, High-performance supercapacitor based on ultralight and elastic three-dimensional carbon foam/reduced graphene/polyaniline nanocomposites, Chinese. Chem. Lett., 30 (2019) 1137-1140.

[17] W. Meng, X. Du, Z. Lin, W. Li, Facile flame deposition of carbon coating onto Ni foam and the study of the derived carbon foam with high capacitive performance, Surf. Coat. Tech., 401 (2020) 126246-126253.

[18] J.C. Caicedo, H.R. Malule, W. Aperador, Mechanical properties evolution in carbon foams obtained from guadua angustifolia, Diam. Relat. Mater., 107 (2020) 107901-107911.

[19] D.C. Marcano, D.V. Kosynkin, J.M. Berlin, A. Sinitskii, Z. Sun, A.S. Slesarev, W. Lu, J.M. Tour, Improved synthesis of graphene oxide, ACS Nano, 4(2010) 4806-4814.

[20] M. Khan, L. Tiehu, T. K. Zhao, Z. Alia, A. Malikb, A. Khan, I. Khan, S. Jiaoa, M. Idrees, C. Xiong, Effect of multi walled carbon nanotubes and diamond nanoparticles on the structure and properties of carbon foams, Diam. Relat. Mater., 79 (2017) 119-126.

[21] S. Li, Y. Song, J. Shi, L. Liu, Q. Guo, Carbon foams with high compressive strength derived from mixtures of mesocarbon microbeads and mesophase pitch, Carbon, 45 (2007) 2092-2097.

[22] S. Elbasuney, G.S.E. Sayyad, M. Yehia, S.K.A. Aal, Facile synthesis of RGO-Fe2O3 nanocomposite: a novel catalyzing agent for composite propellants, J. Mater. Sci.: Mater. Electron., 31 (2020) 20805-20815.

[23] Y.H. Zhao, S.L. Bai, X.W. Yuan, Carbon fibre/graphene foam/polymer composites with enhanced mechanical and thermal properties, Compos. Part B, 94 (2016) 102-108.

[24] J. Ni, R. Zan, J. Qiu, Constructing honeycomb conductive rings in graphene foam/epoxy resin metacomposites for adjustable negative permittivity, low dielectric loss tangent and mechanical enhancement, Org. Electron., 82 (2020) 105706-105712.

[25] K.M. You, S.S. Park, C.S. Lee, J.M. Kim, G.P. Park, W.N. Kim, Preparation and characterization of conductive carbon nanotube-polyurethane foam composites, J. Mater. Sci., 46 (2011) 6850-6855.

[26] S.A. Song, Y.S. Chung, S.S. Kim, The mechanical and thermal characteristics of phenolic foams reinforced with carbon nanoparticles, Compos. Sci. Technol., 103 (2014) 85-93.

[27] L. Zhang, J. Ma, Processing and characterization of syntactic carbon foams containing hollow carbon microspheres, Carbon, 47 (2009) 1451-1456.

[28] D. Irwandi, Z.A. Mas'ud, K. Sutriah, M. Khotib, Preparation and characterization of carbon foam derived from fine coal and phenolic resin, Indones. J. Chem., 16 (2016) 243-248. 
[29] H. Li , Y. Zhang, Y. Li, M. Hu, J. Xu, S. Cao, Synthesis of carbon nanofibers from carbon foam composites via oxyacetylene torch ablation, Mater. Manuf. Process., 30 (2015) 54-58.

[30] L. Jia, M.M. Stevensb, Y. Zhua, Q. Gonga, J. Wua, J. Lianga, Preparation and properties of multi-walled carbon nanotube/carbon/polystyrene composites, Carbon, 47 (2009) 2733-2741.

[31] K. Muhammad, T. Li, Z. Ting Kai, A.A Khurram, K. Imran, U. Azeem, H. Asif, L. Amjad L., A. Farman, I. Sundas, Comparative study of the ball milling and acid treatment of functionalized nanodiamond composites, International Journal of Refractory Metals \& Hard Materials, 73(2018): 46-52.

[32] M. Khan, Li Tiehu, S. B. A. Zaidi, E. Javed, A. Hussain, A. Hayat, A. Zada, D. Alei and A. Ullah, Synergistic effect of nanodiamond and titanium oxide nanoparticles on the mechanical, thermal and electrical properties of pitch-derived carbon foam composites, Polym Int, 2021 DOI 10.1002/pi.6274.

[33] M. Khan, Li Tiehu, A. A. Khurram, T. K. Zhao, C. Xiong, Z. Ali, T. A. Abbas, Asmatullah, I. Ahmad, A. L. Lone, S. Iqbal, A. Khan, Active Sites Determination and De-aggregation of Detonation Nanodiamond Particles, Chiang Mai J. Sci., 44 (2017): 1-14.

[34] M. Khan, N. Shahzad, C. Xiong, T.K. Zhao, T. Li, F. Siddique, N. Ali, M. Shahzad, H. Ullah, S.A. Rakha, Dispersion behavior and the influences of ball milling technique on functionalization of detonated nano-diamonds, Diam. and Relat, Mate. 61 (2016): 32-40.

[35] A. Zada, M. Khan, M. N Qureshi, S. Liu and R. Wang, Accelerating Photocatalytic Hydrogen Production and Pollutant Degradation by Functionalizing g- $\mathrm{C}_{3} \mathrm{~N}_{4}$ With $\mathrm{SnO}_{2}$, Front Chem 7:941 (2020). https://doi.org/10.3389/fchem.2019.00941. 